DEVELOPMENT OF MANAGEMENT

AND ENTREPRENEURSHIP METHODS

ON TRANSPORT, № 2 (75), 2021
РОЗВИТОК МЕТОДІВ

УПРАВЛІННЯ ТА ГОСПОДАРЮВАННЯ

НА ТРАНСПОРТІ, № 2 (75), 2021
УДК 519.86:658.7

JEL M39, O21

DOI 10.31375/2226-1915-2021-2-65-76

МОДЕЛЬ ОПТИМАЛЬНОГО ПЛАНИРОВАНИЯ ПРОИЗВОДСТВА

И ДОСТАВКИ ПРОДУКЦИИ ПОТРЕБИТЕЛЯМ С УЧЕТОМ ЗАТРАТ ПРЕДПРИЯТИЯ НА МАРКЕТИНГ

М.Я. Постан

д.э.н., профессор, заведующий кафедрой «Менеджмент и маркетинг» postan@ukr.net

ORCID: http://orcid.org/00-003-4891-3063?lang=en Ю.В. Куруджи

ст. преподаватель кафедры

«Менеджмент и маркетинг»

Одесский нацчиональный

морской университет, Одесса, Украина

Аннотация. В статье на основе классической модели оптимизации плана выпуска многономенклатурной продукции предприятием и объемов перевозки ее конечным потребителям разработана и проанализирована экономикоматематическая модель совместной оптимизации производственной программы предприятия, доставки готовой продукиии потребителям $u$ маркетинговой деятельности. Предполагается, что спрос на продукцию является некоторой возрастающей функиией от размера затрат на рекламу. Явный вид этой зависимости может быть определен в результате, например, маркетинговых исследований. В частности, она может иметь вид линейной зависимости, при этом ожидаемый спрос на продукцию без учета дополнительных затрат фирмь рекламу считается известным. Модель позволяет определить производственный план предприятия-производителя, планы перевозок для транспортных предприятий, а также размеры затрат на рекламу, максимизируюшие суммарную прибыль предприятия от продажи продукиии с учетом затрат, связанных с производством и доставкой готовой продукиии от предприятияизготовителя в пункты назначения, а также затрат на маркетинг. Предложенный подход может быть использован также для изучения динамических моделей, в которых производственный и перевозочный процессы рассматриваются на заданном горизонте планирования с учетом колебания рыночного спроса.

Ключевые слова: иепь поставок, оптимизачия плана поставок, спрос, маркетинговая деятельность предприятия.
УДК 519.86:658.7

JEL M39, O21

DOI 10.31375/2226-1915-2021-2-65-76

МОДЕЛЬ ОПТИМАЛЬНОГО

ПЛАНУВАННЯ ВИРОБНИЦТВА

ТА ДОСТАВКИ ПРОДУКЦІЇ СПОЖИВАЧАМ

З УРАХУВАННЯМ ВИТРАТ ПІДПРИЕМСТВА НА МАРКЕТИНГ

М.Я. Постан

д.е.н., професор, завідувач кафедри

«Менеджмент і маркетинг» postan@ukr.net

ORCID: http://orcid.org/00-003-4891-3063?lang=en

Ю.В. Куруджи

старший викладач кафедри

«Менеджмент і маркетинг»

yulia.kurudzhi@ukr.net

ORCID: http://orcid.org/00-002-0939-593X

Одеський начіональнии

морський університет, Одеса, Украӥна

Анотація. У статті на основі класично моделі оптимізації плану випуску багатономенклатурної продукиії підприємством $i$ обсягів перевезення ї̈ кінцевим споживачам розроблена та проаналізована економіко-математична модель спільної оптимізачї виробничої програми підприємства, доставки готової продукиії споживачам і маркетингової діяльності. Передбачається, що попит на продукиію $\epsilon$ деякою зростаючою функцією від розміру витрат на рекламу. Явний вигляд иієї залежності може бути визначений в результаті, наприклад, маркетингових досліджень. Зокрема, вона може мати вигляд лінійної залежності, при иьому очікуваний попит на продукиію без урахування додаткових витрат фірми рекламу вважається відомим. Модель дозволяє визначити виробничий план підприємства-виробника, плани перевезень для транспортних підприємств, а також розміри витрат на рекламу, які максимізують сумарний прибуток підприємства від продажу про-дукиіі з урахуванням витрат, пов'язаних 3 виробництвом і доставкою готової продукиії від підприємства-виробника в пункти призначення, а також витрат на маркетинг. Запропонований підхід може бути використаний також для вивчення динамічних моделей, в яких виробничий i перевізний прочеси розглядаються на заданому горизонті планування з урахуванням коливання ринкового попиту.

Ключові слова: ланцңюг поставок, оптимізація планів виробництва та перевезень, попит, маркетингова активність підприємства.

(C) Постан М.Я., Куруджи Ю.В., 2021 
UDC 519.86:658.7

JEL M39, O21

DOI 10.31375/2226-1915-2021-2-65-76

\title{
MODEL OF OPTIMAL OF MANUFACTURING AND DELIVERING OF FINAL PRODUCT TO CONSUMERS TAKING INTO ACCOUNT COST FOR MARKETING
}

\author{
Mykhaylo Postan \\ Doctor of Economics, Professor, Head of the Department of «Management and Marketing» \\ postan@ukr.net \\ ORCID: http://orcid.org/00-003-4891-3063?lang=en
}

\section{Yulia Kurudzhy}

Senior lecturer of the Department of «Management and Marketing» yulia.kurudzhi@ukr.net

ORCID: http://orcid.org/00-002-0939-593X

Odessa National Maritime University, Odessa, Ukraine

\begin{abstract}
The new trends of theory and practice of logistics' development are based on logistical operators and managers of each links of supply chains aspirations to earn the maximal profit from integral paradigm of logistics application based on more close interaction between logistic and marketing strategies of firms or companies. At present, it is very actual the development and analysis of optimization models which are accounting simultaneously influence of marketing activity of companies and the logistics conception. In the article, on the basis of classical model of multi-nomenclature products optimal planning by an enterprise side by side the optimal planning of finished product transportation to consumers the economic-mathematical model has been developed and analyzed taking into account the marketing activity of enterprise. It is supposed that demand for finished products is a some increasing function of advertising costs. The concrete form of such dependence may be found in result of corresponding market's analyses for previous period. In more simple case it may be the linear dependence. It is supposed also that expected demand without cost for advertising is known in result of market analyses. This optimization model allows us to find out the optimal joint production and transportation plans and optimal advertising costs maximizing the total profit of enterprise. The experimental calculations show that interaction of marketing and logistics conceptions allows not only to increase the effectiveness of supply chain functioning as a whole but its every link, as well. The approach proposed may be applied for investigation of more general optimization models taking into account the market demand fluctuation over the given planning horizon. The results obtained in the article show that above optimization model of integrated supply chain and its possible generalizations has the defined perspective of further development in the framework of integral paradigm of logistics.
\end{abstract}

Keywords: supply chain, optimization of the production and transportation plans, demand, marketing activity. 
DEVELOPMENT OF MANAGEMENT

AND ENTREPRENEURSHIP METHODS ON TRANSPORT, № 2 (75), 2021
РОЗВИТОК МЕТОДІВ

УПРАВЛІННЯ ТА ГОСПОДАРЮВАННЯ

НА ТРАНСПОРТІ, № 2 (75), 2021
Постановка проблемы. В настоящее время в теории и практике логистического менеджмента ключевую роль с точки зрения обеспечения эффективной работы как отдельных предприятий, так и цепей поставок в целом играет проблема определения согласованных планов поставок сырья и материалов на производственные предприятия, выпуска готовой продукции и ее доставки конечным потребителям. Поставленные предприятием цели в области маркетинга могут быть достигнуты различными способами, с помощью различных мероприятий. С экономической точки зрения рассматриваемая проблема сводится к обоснованию целесообразности и эффективности дополнительных затрат предприятийизготовителей готовой продукции на разработку и реализацию, например, рекламной компании по сбыту готовой продукции конечным потребителям. Вышесказанное свидетельствует об актуальности рассматриваемой проблемы.

Обзор последних исследований и публикаций. Указанной проблеме взаимодействия логистической и маркетинговой стратегий предприятий посвящено значительное количество публикаций как зарубежных [1-5], так и отечественных авторов [6-9]. Взаимодействие маркетинга и логистики создает возможности повышения эффективности функционирования как всей логистической цепи поставок, так и экономической деятельности предприятия и отдельных его подразделений. Среди работ по исследованию влияния маркетинговой политики на оптимизацию планирования работы цепей поставок можно указать статьи [10-12]. Однако новые тенденции развития логистической теории и практики предполагают стремление логистических операторов и менеджеров отдельных звеньев цепей поставок извлечь максимальную выгоду от использования интегральной парадигмы логистики, используя более тесное взаимодействие логистической и маркетинговой стратегий предприятий, в свете чего нельзя считать рассматриваему проблему окончательно решенной. На наш взгляд, на сегодняшний день исследований на эту тему проводится недостаточно, несмотря на важность проблемы. Актуальным является построение и анализ оптимизационных моделей, учитывающих одновременно маркетинговую деятельность предприятий наряду с использованием логистической концепции цепей поставок, поскольку предоставление логистических услуг должно осуществляться на принципах маркетинга.

Целью данной работы является теоретическое обоснование целесообразности дополнительных затрат на маркетинг для увеличения прибыли предприятия при условии, что в результате маркетинговых исследований известен ожидаемый спрос на продукцию без учета дополнительных затрат фирмы на рекламу.

Основной материал исследования. Продемонстрируем решение указанной проблемы, взяв за основу модель оптимизации плана выпуска многономенклатурной продукции предприятием и объемов перевозки ее конечным потребителям, предложенную в [13].

Рассмотрим некоторое промышленное предприятие, выпускаю- 
щее продукцию $K$ наименований. Производственные издержки по выпуску единицы продукции $k$-го наименования составляют $S_{k}$ (включая затраты на закупку, доставку и хранение сырья). Для выпуска продукции используется $R$ видов сырья, полуфабрикатов и других производственных ресурсов, причем на производство единицы продукции $k$-го вида необходимо затратить $a_{k r}$ сырья $r$-го вида, а ресурс вида $r$ имеется в количестве $b_{r}$.

Произведенная продукция поступает на склад, откуда она должна быть доставлена в $M$ пунктов назначения через $N$ пунктов перевалки, например, через морские порты. Обозначим через $w_{n}$ пропускную способность (или вместимость складов) $n$-го перевалочного пункта, а через $d_{k m}-$ спрос на продукцию $k$-го вида в $m$-м пункте назначения.

Будем считать также, что предприятие на рекламу продажи товара $k$-го вида в $m$-м пункте назначения выделяет денежные средства в количестве $v_{k m}$ с целью увеличения сбыта. В принятых обозначениях это означает, что спрос является некоторой возрастающей функцией от $v_{k m}$, т.e. $d_{k m}=d_{k m}\left(v_{k m}\right)$. Явный вид этой зависимости может быть определен в результате, например, маркетинговых исследований. В частности, она может иметь вид линейной зависимости $d_{k m}\left(v_{k m}\right)=d_{0 k m}\left(1+\alpha v_{k m}\right)$. Здесь множитель $d_{0 k m}$ имеет смысл размера ожидаемого спроса на продукцию $k$-го вида в пункте назначения (потребления) $m$ без учета дополнительных затрат на рекламу, а параметр $\alpha$ определяет степень влияния указанных затрат на увеличение спроca.

Введем вспомогательные множества $B_{k}=\left\{m \mid d_{k m}>0, m=1,2, \ldots, M\right\}$, $k=1,2, \ldots, K$, а также параметры управления рассматриваемой задачи:

$x_{k}$ - количество готовой продукции $k$-го вида, запланированное для выпуска предприятием;

$$
x_{k n} \text { - количество готовой про- }
$$

дукции $k$-го вида, которое планируется для перевалки пункте $P_{n}$;

$$
y_{k n m}-\text { количество готовой про- }
$$
дукции $k$-го вида, которое планируется для доставки из $n$-го перевалочного пункта в $m$-й пункт назначения.

Выражение для суммарной прибыли предприятия от продажи продукции с учетом затрат, связанных с производством и доставкой готовой продукции от предприятия-изготовителя в $M$ пунктов назначения, а также затрат на маркетинг может быть представлено следующим образом:

$$
\begin{aligned}
\Pi_{P}= & \left(\sum_{k=1}^{K} p_{k} x_{k}-\sum_{k=1}^{K} s_{k} x_{k}+\sum_{k=1}^{K} \sum_{n=1}^{N} s_{k n}^{\prime} x_{k n}+\right. \\
& \left.+\sum_{k=1}^{K} \sum_{n=1}^{N} \sum_{m=1}^{M} S_{k n m}^{\prime \prime} y_{k n m}+\sum_{k=1}^{K} \sum_{m=1}^{M} v_{k m}\right),
\end{aligned}
$$

где $p_{k}-$ продажная цена единицы готовой продукции $k$-го вида;

$$
s_{k n}^{\prime} \text { - стоимость перевозки, вклю- }
$$
чая перевалку готовой продукции $k$ го вида в пункт $P_{n}$; 
DEVELOPMENT OF MANAGEMENT
$S_{k n m}^{\prime \prime}-$ стоимость перевозки (включая стоимость погрузки на транспортное средство) единицы продукции $k$-го вида из пункта $P_{n}$ в пункт назначения $D_{m}$.

Ограничения, накладываемые на параметры управления, будут иметь следующий вид:

1) ограничения на производственные ресурсы

$$
\sum_{k=1}^{K} a_{k r} x_{k} \leq b_{r}, r=1,2, \ldots, R
$$

2) вся произведенная продукция должна быть вывезена со склада предприятия

$$
\sum_{n=1}^{N} x_{k n}=x_{k}, k=1,2, \ldots, K
$$

3) потребности всех пунктов назначения должны быть удовлетворены

$$
\sum_{n=1}^{N} y_{k n m}=d_{k m}\left(v_{k m}\right), m \in B_{k}, k=1,2, \ldots, K
$$

4) через пункт перевалки $P_{n}$ не может быть перегружено больше груза, чем его пропускная способность

$$
\sum_{k=1}^{K} \sum_{m \in B_{k}} y_{k n m} \leq w_{n}, n=1,2, \ldots, N
$$

5) вся продукция $k$-го вида, завезенная в $n$-й пункт перевалки, должна быть из него вывезена

$$
x_{k n}=\sum_{m \in B_{k}} y_{k n m}, k=1,2, \ldots, K, n=1,2, \ldots, N \text {; }
$$

6) условия неотрицательности параметров управления

$$
x_{k}, x_{k n}, y_{k n m} \geq 0, \forall k, n, m \text {. }
$$

Таким образом, мы пришли к следующей модели производственнотранспортной задачи: найти производственный план предприятия-производителя $\left\{x_{k}\right\}$, план перевозок для транспортных предприятий $\left\{x_{k n}\right\}$ и $\left\{y_{k n m}\right\}$, а также размеры затрат на рекламу $\left\{v_{k m}\right\}$, которые максимизируют функцию (1) при ограничениях (2)-(7).

Отметим, что из (4), (5) вытекают следующие необходимые условия разрешимости сформулированной задачи оптимизации:

$$
\sum_{k=1}^{K} \sum_{m \in B_{k}} d_{k m}\left(v_{k m}\right) \leq \sum_{n=1}^{N} w_{n} .
$$

Для иллюстрации оптимизационной модели проведем вычисления для нахождения планов перевозки двух видов готовой продукции $(K=2)$, для производства которой используется два вида сырья $(R=2)$. Продукция доставляется в три пункта назначения $(M=3)$ через два пункта перевалки $(N=2)$.

Исходными данными для задачи будут размеры запасов ресурсов для производства $\left(b_{r}\right)$, производственные коэффициенты $\left(a_{k r}\right)$, пропускные способности перевалочных пунктов $\left(w_{n}\right)$, размеры ожидаемого спроса на продукцию без учета дополнительных затрат на рекламу $\left(d_{0 k m}\right)$, коэф- 
фициент, характеризующий степень эффективности рекламы $(\alpha)$, производственные издержки по выпуску единицы продукции $\left(s_{k}\right)$, продажные цены единицы готовой продукции $\left(p_{k}\right)$; стоимости перевозки готовой продукции в пункты перевалки $\left(s_{k n}^{\prime}\right)$ и в пункты назначения $\left(s_{k n m}\right)$.

Необходимые для расчетов значения представлены в табл. 1.

В табл. 2, 3 приведены результаты расчетов, выполненных с помощью пакета Excel для нескольких вариантов исходных данных.

Таблийа 1

Исходные данные для расчета

\begin{tabular}{|c|c|c|c|c|c|}
\hline $\begin{array}{c}\text { Условные } \\
\text { обозначения }\end{array}$ & $\begin{array}{c}\text { Значения } \\
\text { параметров }\end{array}$ & $\begin{array}{c}\text { Условные } \\
\text { обозначения }\end{array}$ & $\begin{array}{c}\text { Значения } \\
\text { параметров }\end{array}$ & $\begin{array}{c}\text { Условные } \\
\text { обозначения }\end{array}$ & $\begin{array}{c}\text { Значения } \\
\text { параметров }\end{array}$ \\
\hline$a_{11}$ & 0,2 & $d_{023}$ & 15 & $s_{113}^{\prime \prime}$ & 2,5 \\
\hline$a_{12}$ & 0,3 & $s_{1}$ & 3,1 & $s_{121}^{\prime \prime}$ & 2,5 \\
\hline$a_{21}$ & 0,3 & $s_{2}$ & 4,1 & $s_{122}^{\prime \prime}$ & 3,1 \\
\hline$a_{22}$ & 0,4 & $p_{1}$ & 10 & $s_{123}^{\prime \prime}$ & 2,8 \\
\hline$w_{1}$ & 120 & $p_{2}$ & 12 & $s_{211}^{\prime \prime}$ & 3,2 \\
\hline$w_{2}$ & 90 & $s_{11}^{\prime}$ & 2,1 & $s_{212}^{\prime \prime}$ & 3,1 \\
\hline$d_{011}$ & 20 & $s_{12}^{\prime}$ & 2,6 & $s_{213}^{\prime \prime}$ & 3,1 \\
\hline$d_{012}$ & 25 & $s_{21}^{\prime}$ & 2,2 & $s_{221}^{\prime \prime}$ & 3,2 \\
\hline$d_{013}$ & 30 & $s_{22}^{\prime}$ & 2,5 & $s_{222}^{\prime \prime}$ & 3,8 \\
\hline$d_{021}$ & 25 & $s_{111}^{\prime \prime}$ & 2,1 & $s_{223}^{\prime \prime}$ & 3,6 \\
\hline$d_{022}$ & 20 & $s_{112}^{\prime \prime}$ & 2,9 & - & - \\
\hline
\end{tabular}

Источник: рассчитано авторами 
Таблийа 2

Результать расчета параметров управления, тыс.т

\begin{tabular}{|c|c|c|c|c|c|c|}
\hline $\begin{array}{c}\text { Условные } \\
\text { обозначения }\end{array}$ & $\begin{array}{l}b_{1}=40 \\
b_{2}=55 \\
\alpha=0,05\end{array}$ & $\begin{array}{l}b_{1}=40 \\
b_{2}=55 \\
\alpha=0,08\end{array}$ & $\begin{array}{l}b_{1}=40 \\
b_{2}=55 \\
\alpha=0,1\end{array}$ & $\begin{array}{l}b_{1}=45 \\
b_{2}=60 \\
\alpha=0,05\end{array}$ & $\begin{array}{l}b_{1}=45 \\
b_{2}=60 \\
\alpha=0,08\end{array}$ & $\begin{array}{l}b_{1}=45 \\
b_{2}=60 \\
\alpha=0,1\end{array}$ \\
\hline 1 & 2 & 3 & 4 & 5 & 6 & 7 \\
\hline$x_{1}$ & 85,0 & 103,33 & 103,33 & 85,0 & 110 & 120 \\
\hline$x_{2}$ & 73,75 & 60 & 60 & 86,25 & 67,50 & 60 \\
\hline$x_{11}$ & 85,0 & 85,0 & 85,0 & 85,0 & 85,0 & 85,0 \\
\hline$x_{12}$ & 0 & 18,33 & 18,33 & 0 & 25,0 & 35,0 \\
\hline$x_{21}$ & 35,0 & 35,0 & 35,0 & 35,0 & 35,0 & 35,0 \\
\hline$x_{22}$ & 38,75 & 25,0 & 25,0 & 51,25 & 32,50 & 25,0 \\
\hline$y_{111}$ & 30 & 48,33 & 48,33 & 30 & 55,0 & 65,0 \\
\hline$y_{112}$ & 25,0 & 6,67 & 6,67 & 25,0 & 0 & 0 \\
\hline$y_{113}$ & 30 & 30 & 30 & 30 & 30 & 20 \\
\hline$y_{121}$ & 0 & 0 & 0 & 0 & 0 & 0 \\
\hline$y_{122}$ & 0 & 18,33 & 18,33 & 0 & 25,0 & 25,0 \\
\hline$y_{123}$ & 0 & 0 & 0 & 0 & 0 & 10 \\
\hline$y_{211}$ & 0 & 0 & 0 & 0 & 0 & 0 \\
\hline$y_{212}$ & 20 & 20 & 20 & 20 & 20 & 20 \\
\hline$y_{213}$ & 15,0 & 15,0 & 15,0 & 15,0 & 15,0 & 15,0 \\
\hline$y_{221}$ & 38,75 & 25,0 & 25,0 & 51,25 & 32,50 & 25,0 \\
\hline$y_{222}$ & 0 & 0 & 0 & 0 & 0 & 0 \\
\hline$y_{223}$ & 0 & 0 & 0 & 0 & 0 & 0 \\
\hline$v_{11}$ & 10,0 & 17,71 & 14,17 & 10,0 & 21,88 & 22,5 \\
\hline$v_{12}$ & 0 & 0 & 0 & 0 & 0 & 0 \\
\hline$v_{13}$ & 0 & 0 & 0 & 0 & 0 & 0 \\
\hline$v_{21}$ & 11,0 & 0 & 0 & 21,0 & 3,75 & 0 \\
\hline$v_{22}$ & 0 & 0 & 0 & 0 & 0 & 0 \\
\hline$v_{23}$ & 0 & 0 & 0 & 0 & 0 & 0 \\
\hline
\end{tabular}

Источник: рассчитано авторами 
Таблийа 3

Результаты расчета прибыли с учетом и без учета затрат на рекламу

\begin{tabular}{|c|c|c|c|c|c|c|}
\hline $\begin{array}{c}\text { Условные } \\
\text { обозначения }\end{array}$ & $\begin{array}{l}b_{1}=40 \\
b_{2}=55 \\
\alpha=0,05\end{array}$ & $\begin{array}{l}b_{1}=40 \\
b_{2}=55 \\
\alpha=0,08\end{array}$ & $\begin{array}{l}b_{1}=40 \\
b_{2}=55 \\
\alpha=0,1\end{array}$ & $\begin{array}{l}b_{1}=45 \\
b_{2}=60 \\
\alpha=0,05\end{array}$ & $\begin{array}{l}b_{1}=45 \\
b_{2}=60 \\
\alpha=0,08\end{array}$ & $\begin{array}{l}b_{1}=45 \\
b_{2}=60 \\
\alpha=0,1\end{array}$ \\
\hline 1 & 2 & 3 & 4 & 5 & 6 & 7 \\
\hline $\begin{array}{l}\text { Затраты на рекламу } \\
\sum_{k=1}^{2} \sum_{n=1}^{3} v_{k n}, \text { тис. ден. ед. }\end{array}$ & 21,0 & 17,71 & 14,17 & 31,0 & 25,62 & 22,5 \\
\hline $\begin{array}{c}\text { Количество произведенной } \\
\text { продукции } \sum_{k=1}^{2} x_{k} \text {, тис.т }\end{array}$ & 158,75 & 163,33 & 163,33 & 171,25 & 177,5 & 180,0 \\
\hline $\begin{array}{c}\text { Прибыль с учетом затрат } \\
\text { на рекламу } \Pi_{P} \text {, тис. ден. ед. }\end{array}$ & 352,75 & 362,46 & 366,0 & 370,25 & 384,38 & 390,0 \\
\hline $\begin{array}{c}\text { Прибыль без учета затрат на } \\
\text { рекламу } \Pi_{Б P} \text {, ден. ед. }\end{array}$ & 319,5 & 319,5 & 319,5 & 319,5 & 319,5 & 319,5 \\
\hline $\begin{array}{c}\Delta \Pi=\Pi_{P}-\Pi_{\text {БP }}, \\
\text { тис. ден. ед. }\end{array}$ & 33,25 & 42,96 & 46,5 & 50,75 & 64,88 & 70,5 \\
\hline
\end{tabular}

Источник: рассчитано авторами

Как видно из табл. 3, рост коэффициента $\alpha$, который характеризует степень эффективности рекламы, приводит к уменьшению затрат на рекламу и увеличению прибыли. При этом увеличивается количество выпускаемой предприятием продукции (при наличии необходимых для производства ресурсов). Увеличение количества сырья, полуфабрикатов и других производственных ресурсов влечет за собой увеличение количества запланированной для производства продукции, рост прибыли и затрат на рекламу (при постоянном значении $\alpha)$ в два раза. Эффективность затрат на рекламу с ростом параметра $\alpha$ иллюстрируется данными о соответ- ствующем росте прибыли, приведенными в заключительной строке табл. 3.

Выводы. Рассмотренная выше модель позволяет дать научно обоснованное согласование производственного плана предприятия-производителя, плана перевозок для транспортных предприятий, а также затрат на рекламу, которые максимизируют суммарную прибыль предприятия от продажи продукции с учетом затрат, связанных с производством и доставкой готовой продукции от предприятия-изготовителя в пункты назначения, включая также затраты на маркетинг. 
В то же время, следует отметить, что такая модель совместной оптимизации производственной программы предприятия, доставки готовой продукции потребителям и маркетинговой деятельности является простейшей статической моделью и может использоваться в логистической практике только с некоторыми оговорками. Поэтому в дальнейшем представляет интерес изучение динамических моделей указанного класса, в которых производственный и перевозочный процессы рассматриваются на заданном горизонте планирования с учетом колебания рыночного спроса
[14], а также нахождение равновесных решений для конкурирующих предприятий-производителей, участвующих в цепях поставок, с учетом маркетинговой активности конкурентов [15].

В целом приведенная экономико-математическая модель интегрированной цепи поставок и отмеченные ее возможные обобщения показывают, что интегральная парадигма логистики имеет определенную перспективу дальнейшего развития.

\section{ЛIТЕРАТУРА}

1. Brandimarte P., Zotteri G. Introduction to Distribution Logistics. NY: J. Wiley\&Sons, Inc., 2007. 579 p.

2. Christopher M., Peck H. Marketing Logistics. Second Edition. Oxford: Elsevier Butterworth-Heinemann, 2003. 168 p.

3. Котлер Ф. Маркетинг-менеджмент. Экспресс-курс, 2-е изд. СПб.: Питер ком. 2006. 464 c.

4. Модели и методы теории логистики: Учебное пособие. 2-е изд. / Под ред. В.С. Лукинского. СПб.: Питер, 2007. 448 с.

5. Ильин И.В., Рыбаков Д.С. Обзор подходов к определению и решению проблемы взаимодействия маркетинга и логистики торгового предприятия // Научно-технические ведомости Санкт-Петербургского государственного политехнического университета. Экономические науки. 2015. № 6 (233). C. 123-137.

6. Dovhun O., Krykavskyy Y. Integration and implementation of marketing and logistics solutions of the enterprises. Przedsiębiorczość i Zarzadzanie. 2019. T. 20, z. 4, cz. 1. S. 103-116.

7. Крикавський С.В. Інтеграиія маркетингу і логістики в системі менеджменту // Вісник Державного університету «Львівська політехніка». 2000. № 416. C. 52-62.

8. Чухрай Н.І., Гірна О.Б. Формування ланџюга поставок: питання теорії $i$ практики. Львів: Інтелект-Захід, 2007. 232 с.

9. Окландер М.А. Маркетинг и логистика в предпринимательстве. О.: АП HТ и ЭИ, 1996. $104 \mathrm{c}$. 
10. Крикавський С.В., Якимишин Л.Я. Компліментарність стратегій маркетингу та логістики в ланцююгу поставок товарів повсякденного попиту // Маркетинг і изифрові технологіі. 2018. Т. 2. № 1. С. 21-32.

11. Клепікова О.А. Вплив маркетингової політики на оптимізацію планування роботи постачальницької фірми в ланџюзі поставок. Вісник Хмельнищького національного університету. Економічні науки. 2020. № 6(288). C. 130-133.

12. Ковальчук С.В., Семенов К.Л. Маркетинг-логістичне забезпечення у ланцңюгах поставок товарів споживчого попиту // Вісник Хмельницького національного університету. Економічні науки. 2017. № 3(1). С. 155-162.

13. Постан М.Я., Малиновский Д.А. Модель оптимального планирования производства и доставки продукичи предприятия по распределительным каналам // Методи та засоби управління розвитком транспортних систем: 3б. наукових працьь ОНМУ. 2009. Bun. 15. C. 19-28.

14. Postan M.Ya., Chuhraj N.I., Kurudzhi Yu.V. Dynamic Model for Optimization of Production and Finished Products Delivery Plans in Supply Chain. Logistyka. 2014. № 4. P. 2345-2352.

15. Kurudzhi Yu., Moskvichenko I., Postan M. Method of finding equilibrium solutions for duopoly of supply chains taking into account the innovation activity of enterprises. Eastern-European Journal of Enterprise Technologies. 2017. № 3/4(87). P. 25-30.

\section{REFERENCES}

1. Brandimarte, P., \& Zotteri, G. (2007). Introduction to Distribution Logistics. NY: J. Wiley\&Sons, Inc., 579.

2. Christopher M., \& Peck H. (2003). Marketing Logistics. Second Edition. Oxford: Elsevier Butterworth-Heinemann, 168.

3. Kotler, F. (2006). Marketing-menedzhment. Ekspress-kurs. [Marketing management. Express Course]. SPb.: Piter kom., 464 [in Russian].

4. Lukinskiy, V.S. (2007). Modeli i metody teorii logistiki: Uchebnoye posobiye. 2-e izd. [Models and methods of logistics theory: A textbook. 2nd ed.]. SPb.: Piter, 448 [in Russian].

5. Il'in, I.V., \& Rybakov, D.S. (2015). Obzor podkhodov k opredeleniyu i resheniyu problemy vzaimodeystviya marketinga i logistiki torgovogo predpriyatiya [Review of approaches to defining and solving the problem of interaction between marketing and logistics of a trading enterprise]. Nauchno-tekhnicheskiye vedomosti Sankt-Peterburgskogo gosudarstvennogo politekhnicheskogo universiteta. Ekonomicheskiye nauki - Scientific and technical statements of the St. Petersburg State Polytechnic University. Economic Sciences, 6(233), 123-137 [in Russian]. 
6. Dovhun, O., \& Krykavskyy, Y. (2019). Integration and implementation of marketing and logistics solutions of the enterprises. Przedsiębiorczość $i$ Zarzadzanie - Entrepreneurship and Management, 20(4/1), 103-116.

7. Krykavskyi, Ye.V. (2000). Intehratsiia marketynhu $i$ lohistyky $v$ systemi menedzhmentu [Integration of marketing and logistics in the management system]. Visnyk Derzhavnoho universytetu «Lvivska politekhnika»-Bulletin of the State University "Lviv Polytechnic», 416, 52-62 [in Ukrainian].

8. Chukhrai, N.I., \& Hirna, O.B (2007). Formuvannia lantsiuha postavok: pytannia teorii i praktyky [Formation of the supply chain: questions of theory and practice]. Lviv: Intelekt-Zakhid, 232 [in Ukrainian].

9. Oklander, M.A. (1996). Marketing i logistika v predprinimatelstve [Marketing and logistics in entrepreneurship]. O.: AP NT i. EI, 104 [in Russian].

10. Krykavsky, Ye.V., \& Yakymyshyn, L.Ia. (2018). Komplimentarnist stratehii marketynhu ta lohistyky $v$ lantsiuhu postavok tovariv povsiakdennoho popytu [Complementarity of marketing and logistics strategies in the supply chain of everyday demand]. Marketynh i tsyfrovi tekhnolohii - Marketing and digital technologies, 2(1), 21-32 [in Ukrainian].

11. Klepikova, O.A. (2020). Vplyv marketynhovoi polityky na optymizatsiiu planuvannia roboty postachalnytskoi firmy $v$ lantsiuzi postavok [On influence of supply firms market policy on optimization of its ordering policy with supply chain]. Visnyk Khmelnytskoho natsionalnoho universytetu. Ekonomichni nauky - Bulletin of Khmelnytsky National University. Economic sciences, 6(288), 130-133 [in Ukrainian].

12. Kovalchuk, S.V., Semenov, K.L. (2017). Marketynh-lohistychne zabezpechennia u lantsiuhakh postavok tovariv spozhyvchoho popytu [Marketing and logistics in supply chains of consumer demand].Visnyk Khmelnytskoho natsionalnoho universytetu. Ekonomichni nauky - Bulletin of Khmelnytsky National University. Economic sciences, 3(1), 155-162 [in Ukrainian].

13. Postan, M.Ya., \& Malinovskiy, D.A. (2009). Model optimal'nogo planirovania proizvodstva i dostavki produktsii predpriatia po raspredelitel'nym kanalam [Model of optimal planning of production and delivery of product by distributive channels]. Metody ta zasoby upravlinnia rozvytkom transportnyh system. Zbirnyk naukovyh pratz Odes'kogo national'noho universytetu Collection of scientific papers of Odessa National Maritime University, 15, 19-28 [in Russian].

14. Postan M.Ya., Chuhraj, N.I., \& Kurudzhi, Yu.V. (2014). Dynamic Model for Optimization of Production and Finished Products Delivery Plans in Supply Chain. Logistyka, 4, 2345-2352. 
15. Kurudzhi, Yu., Moskvichenko, I., \& Postan, M. (2017). Method of finding equilibrium solutions for duopoly of supply chains taking into account the innovation activity of enterprises. Eastern-European Journal of Enterprise Technologies. 3/4(87). P. 25-30.

Стаття надійшла 24.05.2021

Reference a JournalArtic: Postan, Mykhaylo \& Kurudzhy, Yulia (2021). Model of Optimal of manufacturing and Delivering of Final Product to Consumers Taking into Account Cost for Marketing. Development of management and entrepreneurship methods on transport. 2 (75), 65-76. DOI 10.31375/2226-1915-2021-2-65-76.

Article received 24.05.2021

Посилання на статтю: Постан М.Я., Куруджи Ю.В. Модель оптимального планування виробництва та доставки продукції споживачам 3 урахуванням витрат підприємства на маркетинг // Розвиток методів управління та господарювання на транспорті: Зб. наук. праць, 2021. № 2 (75). C. 65-76. DOI 10.31375/2226-1915-2021-2-65-76. 